\title{
Erratum to: Asymmetric Periodic Solutions in the Restricted Problem of Three Bodies
}

\author{
K. E. Papadakis • M. I. Rodi
}

Published online: 17 February 2010

(C) Springer Science+Business Media B.V. 2010

\section{Erratum to: Earth Moon Planet (2010) 106:37-53 DOI 10.1007/s11038-009-9345-4}

In our effort to extend our present results on the asymmetric periodic orbits in the restricted three-body problem, we found that the results in this paper concerning the case of the Earth-Moon mass distribution (subsection 2.2) are not correct.

The equations of motion of the restricted problem have the symmetry property, with respect to the $x$-axis, that if $x=x(t), y=y(t)$ is a particular solution, then $x=x(-t)$, $y=-y(-t)$ is also a solution (see Szebehely 1967, p. 426). The corresponding symmetry property, with respect to $y$-axis, is also true in the case where the two primary bodies are equal, i.e. $\mu=0.5$ (subsection 2.1 in our results) but it is not true for $\mu \neq 0.5$ (subsection 2.2 in our results).

So the results of subsection 2.2 should be ignored. The numerical determination of the asymmetric periodic orbits in this case $(\mu \neq 0.5)$, needs a different procedure which we are applying at this time and will present in a future article.

\section{Reference}

V. Szebehely, Theory of Orbits (Academic Press, New York, 1967)

The online version of the original article can be found under doi:10.1007/s11038-009-9345-4.

K. E. Papadakis $(\bowtie) \cdot$ M. I. Rodi

Department of Engineering Sciences, University of Patras, 26504 Patras, Greece e-mail: k.papadakis@des.upatras.gr

M. I. Rodi

e-mail: marrod@upatras.gr 